\title{
Statistical analysis of transfer of fluctuations in solar wind turbulence
}

\author{
M. Strumik ${ }^{1}$ and W. M. Macek ${ }^{1,2}$ \\ ${ }^{1}$ Space Research Centre, Polish Academy of Sciences, Bartycka 18 A, 00-716 Warsaw, Poland \\ ${ }^{2}$ Faculty of Mathematics and Natural Sciences, Cardinal Stefan Wyszyński University, Dewajtis 5, 01-815 Warsaw, Poland
}

Received: 17 March 2008 - Revised: 2 July 2008 - Accepted: 2 July 2008 - Published: 28 July 2008

\begin{abstract}
We present results of statistical analysis of the transfer of fluctuations in solar wind turbulence. We investigate the dynamics of the slow solar wind using an approach based on the Markov processes theory and experimental data measured by ACE spacecraft. In particular, we test whether the Chapman-Kolmogorov equation is approximately satisfied for the turbulent cascade. We consider the following cases of transfer of fluctuations: magnetic-to-magnetic, velocity-to-velocity, velocity-to-magnetic, and magnetic-tovelocity. In all these cases, the obtained results confirm local character of the transfer of fluctuations.
\end{abstract}

\section{Introduction}

In the plasma flow expanding from the Sun into the interplanetary space we can distinguish several forms, in particular the slow $(<450 \mathrm{~km} / \mathrm{s})$ and fast $(>600 \mathrm{~km} / \mathrm{s})$ solar wind (see, e.g., Schwenn $(1990,2006)$ and references therein). The fast wind is more homogeneous and incompressible in comparison with the slow wind, but both types of the solar wind are quite variable in space and time. In fact, irregular dynamics of the solar wind plasma exhibits many similarities to fully developed hydrodynamic turbulence. Numerous in situ measurements of temporal variability of parameters of the plasma have shown that their spectral distributions usually have power-law character (Matthaeus and Goldstein, 1982; Goldstein et al., 1995; Tu and Marsch, 1995; Goldstein and Roberts, 1999). Investigations of the fluctuations have also revealed that the shape of their probability distributions changes from Gaussian at large scales to strongly non-Gaussian (heavy-tailed) at small scales, which is commonly attributed to intermittency phenomenon (Marsch and

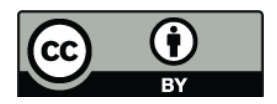

Correspondence to: M. Strumik (maro@cbk.waw.pl)
Tu, 1994; Sorriso-Valvo et al., 1999, 2001; Burlaga, 2001), which is related to multifractality (Burlaga, 1991; Marsch et al., 1996; Macek and Szczepaniak, 2008). In fact, the solar wind provides a unique laboratory for studying highReynolds-number magnetohydrodynamic turbulence (see, e.g., Goldstein et al. (1995); Bruno and Carbone (2005) for review).

Recently, a great deal of attention has been devoted to investigations of the fluctuations in hydrodynamic turbulence from the point of view of the theory of Markov processes (see, e.g., Pedrizzetti and Novikov, 1994; Friedrich and Peinke, 1997a,b; Davoudi and Tabar, 1999; Renner et al., 2001). In particular, results of the verification of the validity of the Chapman-Kolmogorov equation as well as the estimation of the Kramers-Moyal coefficients from experimental data suggest that the Markov processes approach may be appropriate to the description and modeling of the turbulent cascade (Friedrich and Peinke, 1997a,b; Renner et al., 2001). The estimation of the Kramers-Moyal coefficients allows to determine the form of the Fokker-Planck equation governing the evolution of the probability distribution with scale for the fluctuations. A model based on the Fokker-Planck equation has been recently proposed for solar wind turbulence, but only for fluctuations of quantities that exhibit self-similar scaling (Hnat et al., 2003).

In this paper we use the approach based on the Markov processes theory to examine the character of the transfer of fluctuations between different scales in solar wind turbulence. In particular, we analyze whether this transfer is local or nonlocal. The notion of locality used in this paper does not refer to locality in physical space. Here we use the words "local" and "nonlocal" in terms of the scales involved in interactions leading to the turbulent cascade. If the interaction involves scales (eddies) of similar size it is local, otherwise it is nonlocal. The question of locality of the energy transfer is of some interest, e.g., in the studies of dynamo mechanism to generate magnetic fields in astrophysical objects, where

Published by Copernicus Publications on behalf of the European Geosciences Union and the American Geophysical Union. 
nonlocal processes of the generation of large-scale fields by small-scale helicities in helical MHD turbulence are studied in details (see, e.g., Sect. 6.2.1 of Biskamp, 2003). This question is also important for modeling MHD flows and numerical simulations. For example, this is essential in largeeddy simulations, where low-pass filtering with respect to a cutoff wave number requires some assumptions concerning the transfer of energy around the cutoff wavenumber. Local and nonlocal transfer mechanisms can be distinguished in theoretical studies of turbulence by shell models or numerical simulations (see, e.g., Alexakis et al., 2005; Debliquy et al., 2005; Mininni et al., 2005; Verma et al., 2005), but it is difficult to study the property of turbulence directly using experimental data. Here we argue that a method of statistical analysis based on the Markov processes theory provides a tool that allows to distinguish between local and nonlocal transfer mechanisms in an experimental situation.

This paper is organized as follows. In Sect. 2 we describe data set used for analysis. Then we present the Markov processes approach (Sect. 3) and its physical interpretation (Sect. 4). In Sect. 5 we present results of our analysis, which are then discussed and summarized in Sect. 6.

\section{Data set}

We analyze the measurements of the magnitudes of the proton bulk velocity and magnetic field obtained by the ACE spacecraft from 1999 to 2006 . In this paper we focus on the slow solar wind, and the data set contains measurements from interleaved streams of the slow and fast solar wind. Therefore, from all the data available we have chosen subintervals of the length of two days or more, where the average bulk velocity of the solar wind is never above $450 \mathrm{~km} / \mathrm{s}$. Since the magnetic field data are provided at time resolution of $16 \mathrm{~s}$, they are linearly interpolated to the grid of time resolution of $64 \mathrm{~s}$, which is used for the velocity data. We use the linear interpolation instead of decimating the magnetic field data because in the case of ACE spacecraft some slight shifts of time grid occur sometimes. The linear interpolation allows us to shift safely the time grid for the magnetic field data to the time grid for the velocity data. The data set analyzed here consists of about $10^{6}$ measurements obtained in the ecliptic plane in the proximity of L1 libration point at radial distance from the Sun of 0.99 AU.

Further we consider fluctuations of the magnetic field defined as $b(t)=B(t+\tau)-B(t)$, where $B(t)$ is the magnitude of the magnetic field measured by the MAG instrument of the ACE spacecraft (Smith et al., 1998). Similarly, fluctuations of the magnitude of the bulk velocity of plasma are defined as $v(t)=V(t+\tau)-V(t)$, where $V(t)$ is the magnitude of the velocity measured by the SWEPAM instrument (McComas et al., 1998). Presenting our results we use here temporal scales and do not recast the fluctuations into the space domain using the Taylor hypothesis. However, one should bear in mind that we analyze highly supersonic and super-Alfvénic flow (mean velocity $U$ of about $400 \mathrm{~km} / \mathrm{s}$ in the reference system moving with the measuring instrument). Hence physically the measured temporal scales are rather related to spatial scales. Assuming that the Taylor hypothesis is satisfied here, one can easily transform the temporal scale $\tau$ to the spatial scale $l$ using the relationship $l=U \tau$ (Frisch, 1995). However, in general it is not possible to distinguish between temporal and spatial variations in the case of onepoint measurements of plasma parameters, as it is for the ACE spacecraft.

\section{Markov processes approach}

We investigate here statistics of fluctuations $x(t)=X(t+\tau)-X(t)$ of a physical quantity $X(t)$ at a scale $\tau$. We consider the fluctuations as a stochastic process in scale, i.e., we assume that a turbulent cascade is responsible for the transfer of a fluctuation $x_{i}$ at the largest (energy-containing) scale $\tau_{i}$ to a fluctuation $x_{i-1}$ at a smaller scale $\tau_{i-1}$, then the fluctuation $x_{i-1}$ at the scale $\tau_{i-1}$ to a fluctuation $x_{i-2}$ at a scale $\tau_{i-2}$, and so forth till dissipation scale is reached. Using the joint probability density $P\left(x_{1}, \tau_{1} ; x_{2}, \tau_{2}\right)$ of finding the fluctuations $x_{1}$ at a scale $\tau_{1}$ and $x_{2}$ at a scale $\tau_{2}$, where $\tau_{1}<\tau_{2}$, we can define the conditional p.d.f. as $P\left(x_{1}, \tau_{1} \mid x_{2}, \tau_{2}\right)=P\left(x_{1}, \tau_{1} ; x_{2}, \tau_{2}\right) / P\left(x_{2}, \tau_{2}\right)$. By analogy to the definition of the two-point probability distributions, one can define the joint and conditional probability densities for longer sequences of fluctuations $x_{1}, x_{2}, x_{3}, \ldots$ at scales $\tau_{1}, \tau_{2}, \tau_{3}, \ldots$. In the case of a Markov process, by definition the following condition must be satisfied

$P\left(x_{1}, \tau_{1} \mid x_{2}, \tau_{2} ; \ldots ; x_{N}, \tau_{N}\right)=P\left(x_{1}, \tau_{1} \mid x_{2}, \tau_{2}\right)$,

thus the $N$-point joint probability distribution function (p.d.f.) $\quad P\left(x_{1}, \tau_{1} ; x_{2}, \tau_{2} ; \ldots ; x_{N}, \tau_{N}\right)$ is determined by the product of conditional probabilities $P\left(x_{i-1}, \tau_{i-1} \mid x_{i}, \tau_{i}\right)$, where $\tau_{i-1}<\tau_{i}$. For a finite set of experimental data, the Markov property can be verified by comparison of a conditional p.d.f. $P_{\mathrm{E}}\left(x_{1}, \tau_{1} \mid x_{2}, \tau_{2}\right)$ evaluated directly from data with the p.d.f. computed using the Chapman-Kolmogorov equation

$$
\begin{aligned}
& P\left(x_{1}, \tau_{1} \mid x_{2}, \tau_{2}\right)= \\
& =\int_{-\infty}^{\infty} P\left(x_{1}, \tau_{1} \mid x^{\prime}, \tau^{\prime}\right) P\left(x^{\prime}, \tau^{\prime} \mid x_{2}, \tau_{2}\right) \mathrm{d} x^{\prime},
\end{aligned}
$$

where $\tau_{1}<\tau^{\prime}<\tau_{2}$ (see Figs. 1 or 2 for illustration).

Equation (2) is a necessary condition for a stochastic process to be Markovian. The Chapman-Kolmogorov equation can be written in a differential form using the so-called Kramers-Moyal expansion

$$
\begin{aligned}
& -\tau \frac{\partial P\left(x, \tau \mid x_{0}, \tau_{0}\right)}{\partial \tau}= \\
& =\sum_{k=1}^{\infty}\left(-\frac{\partial}{\partial x}\right)^{k} D^{(k)}(x, \tau) P\left(x, \tau \mid x_{0}, \tau_{0}\right) .
\end{aligned}
$$




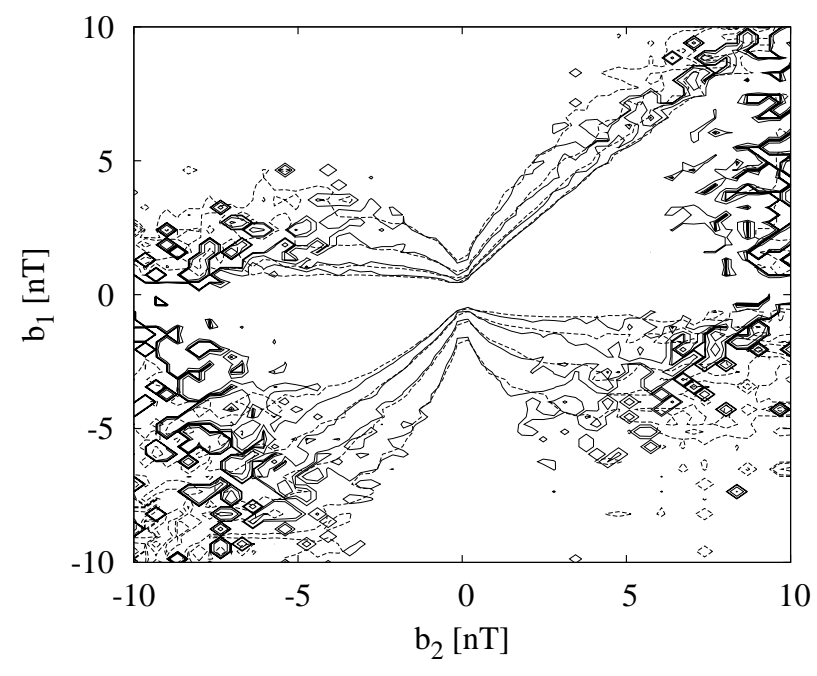

Fig. 1. Contour plots illustrating verification of the ChapmanKolmogorov equation for magnetic field fluctuations. Solid lines represent the conditional p.d.f. $P_{\mathrm{E}}\left(b_{1}, \tau_{1} \mid b_{2}, \tau_{2}\right)$ evaluated directly from data, whereas dashed lines show the conditional p.d.f. $P\left(b_{1}, \tau_{1} \mid b_{2}, \tau_{2}\right)$ computed using Eq. (2). The subsequent isolines correspond to the following levels of the p.d.f.: $0.05,0.02,0.005,0.001$ (from the middle of the plot).

Kramers-Moyal coefficients $D^{(k)}(x, \tau)$ can be evaluated by the limit $\Delta \tau \rightarrow 0$ of the conditional moments $M^{(k)}(x, \tau, \Delta \tau)$, namely

$D^{(k)}(x, \tau)=\lim _{\Delta \tau \rightarrow 0} M^{(k)}(x, \tau, \Delta \tau)$

and

$M^{(k)}(x, \tau, \Delta \tau)=$

$=\frac{\tau}{k ! \Delta \tau} \int_{-\infty}^{\infty}\left(x^{\prime}-x\right)^{k} P\left(x^{\prime}, \tau^{\prime} \mid x, \tau\right) \mathrm{d} x^{\prime}$,

where $\Delta \tau=\tau-\tau^{\prime}$. In comparison with the definition used by Risken (1989), the conditional moments given in Eq. (5) and the resulting Kramers-Moyal coefficients in Eq. (4) are multiplied by $\tau$, which is equivalent to a logarithmic length scale (Renner et al., 2001).

As shown by Friedrich and Peinke (1997a,b), it is possible to estimate the Kramers-Moyal coefficients from experimental data. In particular, if the estimated coefficient $D^{(4)}(x, \tau)$ vanishes, then according to the Pawula theorem: $D^{(k)}(x, \tau)=0$ for $k \geq 3$ (Risken, 1989). In this case, starting from Eq. (3) we arrive at the Fokker-Planck equation

$-\tau \frac{\partial P(x, \tau)}{\partial \tau}=$
$=\left(-\frac{\partial D^{(1)}(x, \tau)}{\partial x}+\frac{\partial^{2} D^{(2)}(x, \tau)}{\partial x^{2}}\right) P(x, \tau)$,

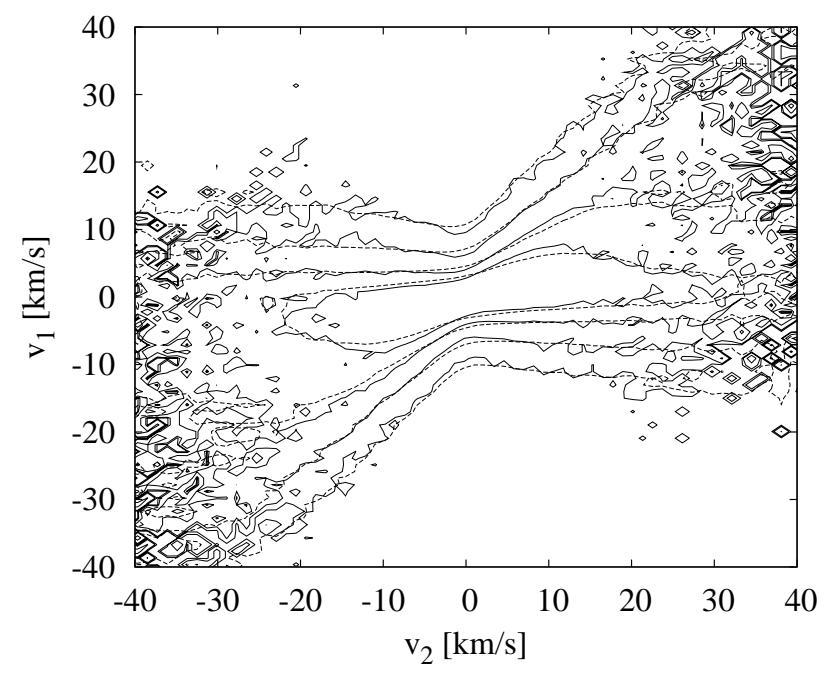

Fig. 2. Contour plots illustrating verification of the ChapmanKolmogorov equation for velocity fluctuations. Solid lines represent the conditional p.d.f. $P_{\mathrm{E}}\left(v_{1}, \tau_{1} \mid v_{2}, \tau_{2}\right)$ evaluated directly from data, whereas dashed lines show the conditional p.d.f. $P\left(v_{1}, \tau_{1} \mid v_{2}, \tau_{2}\right)$ computed using Eq. (2). The subsequent isolines correspond to the following levels of the p.d.f.: $0.05,0.02,0.005,0.001$ (from the middle of the plot).

which determines the evolution of the probability distribution function of a stochastic process with scale $\tau$. Therefore, by estimating the coefficients $D^{(1)}(x, \tau)$ and $D^{(2)}(x, \tau)$ from experimental data, one should be able to model experimentally observed collapse of the probability distribution of fluctuations with changing scale for a turbulent cascade.

\section{Physical consequences of Markovian character of tur- bulence}

If Eq. (2) is satisfied, then the transition probability from scale $\tau_{2}$ to $\tau_{1}$ can be divided into transitions from $\tau_{2}$ to $\tau^{\prime}$ and then from $\tau^{\prime}$ to $\tau_{1}$. Therefore, in the case of a turbulent cascade, the fulfillment of the Chapman-Kolmogorov equation for all triplets $\tau_{1}<\tau^{\prime}<\tau_{2}$ in the inertial range suggests the presence of a local transfer mechanism in the cascade. If we consider fluctuations in time domain, it means that the transfer process is local in scale. However, if the root-meansquare of the velocity fluctuations is small as compared to the mean velocity of the flow (Taylor's hypothesis), temporal variations at a fixed position are interpreted as spatial variations. In this case, the local transfer in scale can be interpreted as directly related to the local transfer in wave vector space.

Typically, in MHD turbulence we observe strong interactions between velocity and magnetic field modes, giving input to the transfer of energy between these quantities. These interactions should be seen as a statistical dependence 

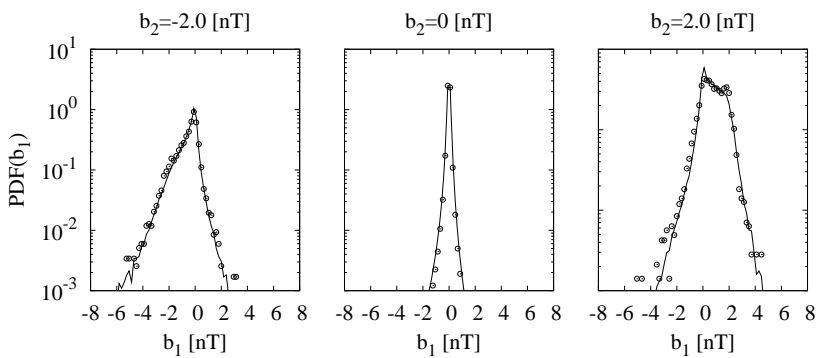

Fig. 3. Verification of the Chapman-Kolmogorov Eq. (2) for magnetic field fluctuations. Comparison of cuts through $P_{\mathrm{E}}\left(b_{1}, \tau_{1} \mid b_{2}, \tau_{2}\right)$ (points) and $P\left(b_{1}, \tau_{1} \mid b_{2}, \tau_{2}\right)$ (lines) from Fig. 1 are shown for fixed values of $b_{2}$, namely $b_{2}=-2 \mathrm{nT}$ (on the left), $b_{2}=0 \mathrm{nT}$ (in the middle), and $b_{2}=2 \mathrm{nT}$ (on the right).
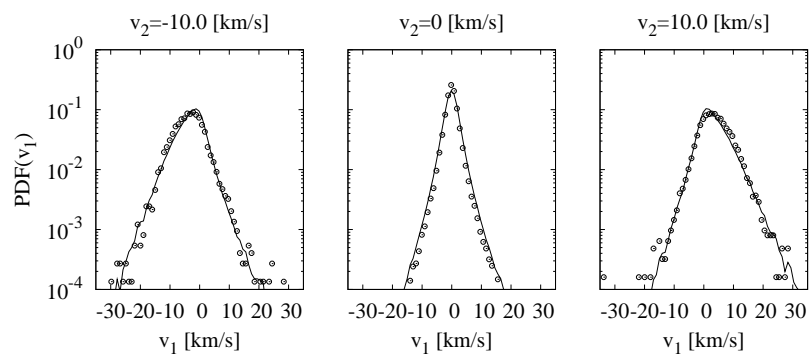

Fig. 4. Verification of the Chapman-Kolmogorov Eq. (2) for velocity fluctuations. Comparison of cuts through $P_{\mathrm{E}}\left(v_{1}, \tau_{1} \mid v_{2}, \tau_{2}\right)$ (points) and $P\left(v_{1}, \tau_{1} \mid v_{2}, \tau_{2}\right)$ (lines) from Fig. 2 are shown for fixed values of $v_{2}$, namely $v_{2}=-10 \mathrm{~km} / \mathrm{s}$ (on the left), $v_{2}=0 \mathrm{~km} / \mathrm{s}$ (in the middle), and $v_{2}=10 \mathrm{~km} / \mathrm{s}$ (on the right).

between fluctuations of the magnetic field $b(t)$ and velocity $v(t)$. In Eq. (2) we assume that the transfer of fluctuations is related to the same quantity $x$ for all three scales $\tau_{1}, \tau^{\prime}, \tau_{2}$. To take into account transfer of fluctuations between different quantities, we propose the following modification of Eq. (2)

$P\left(x_{1}, \tau_{1} \mid y_{2}, \tau_{2}\right)=$

$=\int_{-\infty}^{\infty} P\left(x_{1}, \tau_{1} \mid y^{\prime}, \tau^{\prime}\right) P\left(y^{\prime}, \tau^{\prime} \mid y_{2}, \tau_{2}\right) \mathrm{d} y^{\prime}$.

By analogy with Eq. (2), Eq. (7) should allow to examine whether the transfer of fluctuations between the two quantities $y$ and $x$ has local or nonlocal character. Namely, if the conditional p.d.f. $P_{\mathrm{E}}\left(x_{1}, \tau_{1} \mid y_{2}, \tau_{2}\right)$ evaluated directly from data overlaps with the p.d.f. computed from Eq. (7), then the transfer of fluctuations can be subdivided into smaller steps and one can infer that the transfer has local character in scale.

Instead of Eq. (7) one could use a definition containing $\int_{-\infty}^{\infty} P\left(x_{1}, \tau_{1} \mid x^{\prime}, \tau^{\prime}\right) P\left(x^{\prime}, \tau^{\prime} \mid y_{2}, \tau_{2}\right) \mathrm{d} x^{\prime}$, where the quantity for intermediate scale is different from that in Eq. (7). We

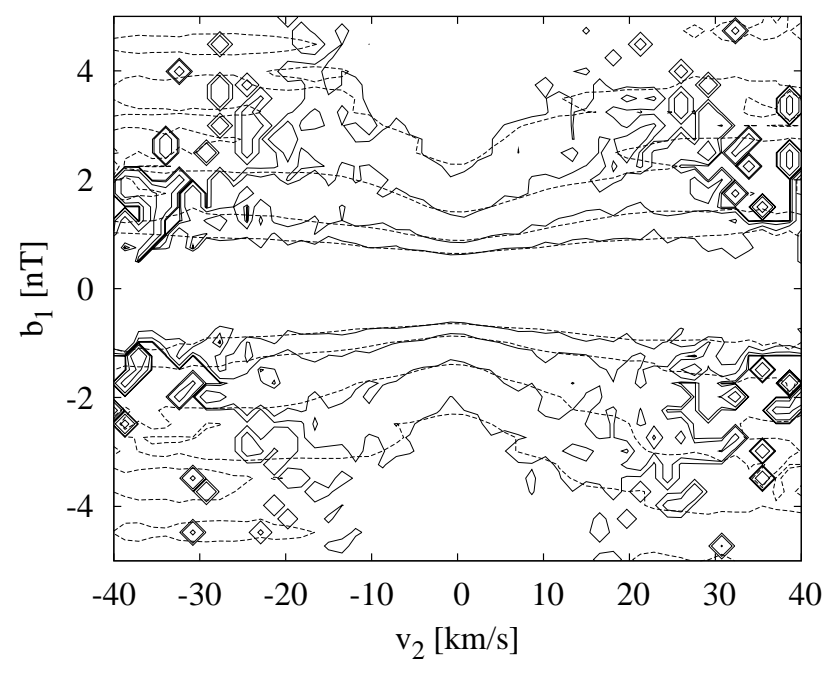

Fig. 5. Contour plots illustrating verification of the modified Chapman-Kolmogorov equation for velocity-to-magnetic transfer of fluctuations. Solid lines represent the conditional p.d.f. $P_{\mathrm{E}}\left(b_{1}, \tau_{1} \mid v_{2}, \tau_{2}\right)$ evaluated directly from data, whereas dashed lines show the conditional p.d.f. $P\left(b_{1}, \tau_{1} \mid v_{2}, \tau_{2}\right)$ computed using Eq. (7). The subsequent isolines correspond to the following levels of the p.d.f.: $0.05,0.02,0.005,0.001$ (from the middle of the plot).

have checked these two definitions in our computations and they generate very similar results, thus further in our paper we present only results obtained from Eq. (7).

\section{Results}

In Figs. 1 and 2 we show superposed contour plots of the conditional p.d.f. estimated directly from data (solid lines) and the p.d.f. computed using Eq. (2) (dashed lines) for $\tau_{1}=64$, $\tau^{\prime}=128$, and $\tau_{2}=192$ seconds. The plots have been obtained for magnetic-to-magnetic and velocity-to-velocity transfer of fluctuations, correspondingly. In Figs. 3 and 4 we additionally show cuts through the conditional probability distributions shown in Figs. 1 and 2 for fixed values of $b_{2}$ and $v_{2}$, respectively.

In Figs. 5 and 6 we present superposed contour plots of the conditional p.d.f. estimated directly from data (solid lines) and the p.d.f. computed using Eq. (7) (dashed lines) for $\tau_{1}=64, \tau^{\prime}=128$, and $\tau_{2}=192$ seconds. The plots have been obtained for velocity-to-magnetic and magnetic-to-velocity transfer of fluctuations, correspondingly. In Figs. 7 and 8 we additionally show cuts through the conditional probability distributions shown in Figs. 5 and 6 for fixed values of $v_{2}$ and $b_{2}$, respectively.

One can see that corresponding contour lines for the experimental and computed probability distributions are very close to each other in the central part of Figs. 1, 2, 5, and 


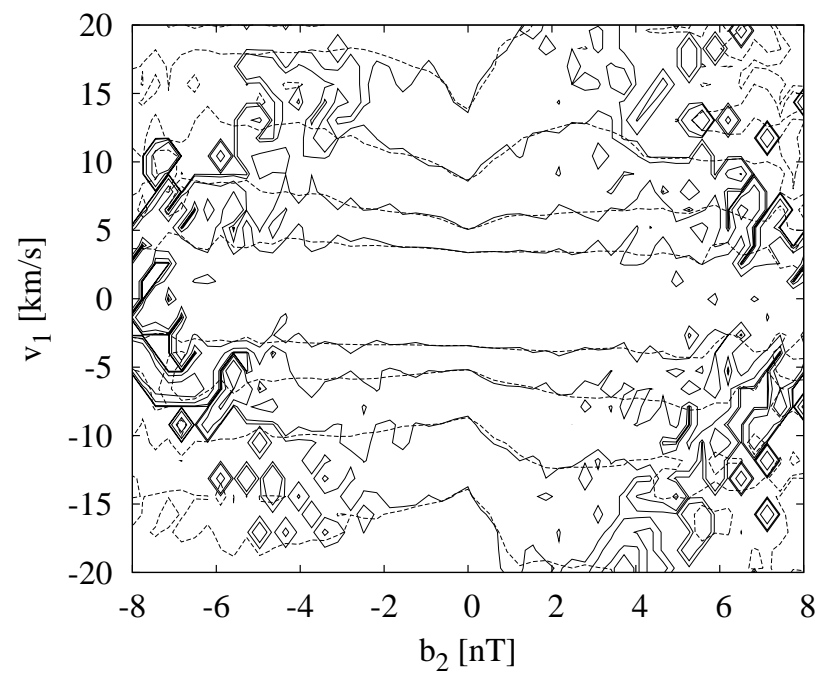

Fig. 6. Contour plots illustrating verification of the modified Chapman-Kolmogorov equation for magnetic-to-velocity transfer of fluctuations. Solid lines represent the conditional p.d.f. $P_{\mathrm{E}}\left(v_{1}, \tau_{1} \mid b_{2}, \tau_{2}\right)$ evaluated directly from data, whereas dashed lines show the conditional p.d.f. $P\left(v_{1}, \tau_{1} \mid b_{2}, \tau_{2}\right)$ computed using Eq. (7). The subsequent isolines correspond to the following levels of the p.d.f.: $0.05,0.02,0.005,0.001$ (from the middle of the plot).

6. In the periphery of the plots there are strong irregularities resulting from small number of counts during the estimation of the probability distributions, thus direct verification of the applicability of Eqs. (2) or (7) is not possible in this region. In Figs. 3, 4, 7, and 8 points representing cuts through experimental p.d.f. fit rather well the lines representing cuts through computed p.d.f. The obtained results indicate that the Chapman-Kolmogorov Eq. (2) (or its modified version (7), where appropriate) is (at least approximately) satisfied for the range of scales from $\tau_{1}=64$ to $\tau_{2}=192 \mathrm{~s}$, for all of the examined transfers of fluctuations.

\section{Conclusions}

Our results suggest that the Chapman-Kolmogorov Eq. (2) (or its modified version (7), where appropriate) is approximately satisfied for the smallest scales available for testing here (tens or hundreds of seconds). In fact we have repeated the computations also for larger scales (the results are not shown here) verifying that the Chapman-Kolmogorov equation is satisfied for scales up to about twenty hours. Therefore, our results show that the Markov processes approach can be applied to the description of the turbulent cascade in slow solar wind turbulence. These results confirm the universality of the statistical approach to the description of a turbulent cascade proposed by Friedrich and Peinke (1997a,b) and based on the Markov processes theory.
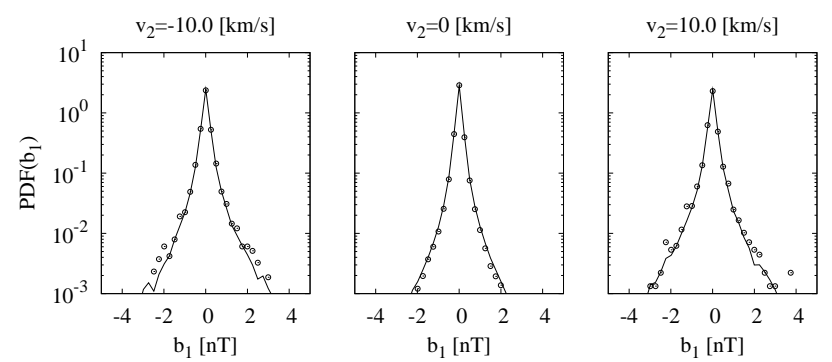

Fig. 7. Verification of the modified Chapman-Kolmogorov Eq. (7) for velocity-to-magnetic transfer of fluctuations. Comparison of cuts through $P_{\mathrm{E}}\left(b_{1}, \tau_{1} \mid v_{2}, \tau_{2}\right)$ (points) and $P\left(b_{1}, \tau_{1} \mid v_{2}, \tau_{2}\right)$ (lines) from Fig. 5 are shown for fixed values of $v_{2}$, namely $v_{2}=-10 \mathrm{~km} / \mathrm{s}$ (on the left), $v_{2}=0 \mathrm{~km} / \mathrm{s}$ (in the middle), and $v_{2}=10 \mathrm{~km} / \mathrm{s}$ (on the right).

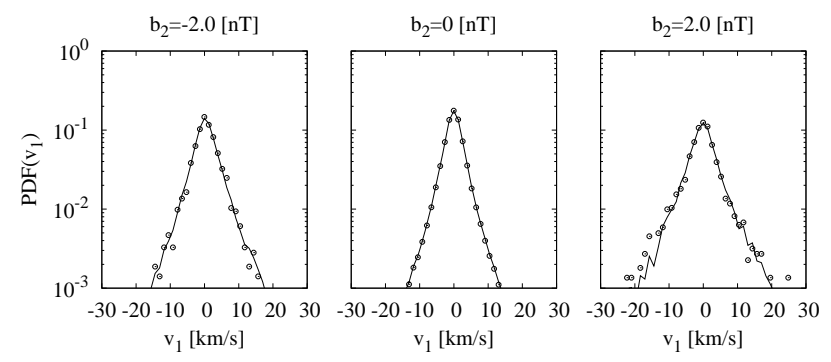

Fig. 8. Verification of the modified Chapman-Kolmogorov Eq. (7) for magnetic-to-velocity transfer of fluctuations. Comparison of cuts through $P_{\mathrm{E}}\left(v_{1}, \tau_{1} \mid b_{2}, \tau_{2}\right)$ (points) and $P\left(v_{1}, \tau_{1} \mid b_{2}, \tau_{2}\right)$ (lines) from Fig. 6 are shown for fixed values of $b_{2}$, namely $b_{2}=-2 \mathrm{nT}$ (on the left), $b_{2}=0 \mathrm{nT}$ (in the middle), and $b_{2}=2 \mathrm{nT}$ (on the right).

In isotropic hydrodynamical turbulence larger eddies only advect smaller eddies without altering their scale significantly, thus mainly interactions between eddies of similar size give input to the energy cascade. Therefore, the assumption of the locality of the energy transfer in wave vector space is well justified, although some details are still not understood completely (see, e.g., Kishida et al., 1999). On the contrary, the question of locality of the energy transfer in MHD turbulence is much less understood and more complicated because of strong influence of the mean magnetic field on plasma dynamics. According to the classical IroshnikovKraichnan picture, taking into account the Alfvén effect, we should expect nonlocal influence of large-scale magnetic field on small-scale turbulent eddies, and so also nonlocal interactions between modes (Pouquet et al., 1976; Biskamp, 2003). However, results of recent numerical simulations suggest that local transfer mechanisms dominate in MHD turbulence (Debliquy et al., 2005; Alexakis et al., 2005; Mininni et al., 2005). 
Local and nonlocal transfer mechanisms can be distinguished in theoretical studies of turbulence using shell models or numerical simulations, but it is difficult to study the property of turbulence directly using experimental data. The Markov processes approach seems to provide such a method. Namely, analyzing a time series measured in a turbulent flow we should be able to identify the character of the dominating transfer mechanism for a given quantity or between different quantities, i.e., we should be able to answer the question as to whether the mechanism is local or nonlocal in scale. As we argued in Sect. 4, in the case of turbulent cascade fulfillment of Eqs. (2) or (7) suggests the presence of a local transfer mechanism in scale, which means that it is local in the wave vector space, provided that the Taylor hypothesis is satisfied.

Our results indicate that the Chapman-Kolmogorov Eq. (2) is approximately satisfied for velocity-to-velocity and magnetic-to-magnetic transfers of fluctuations, whereas its modified version (7) is satisfied for velocity-to-magnetic and magnetic-to-velocity transfers. This suggests that the local transfer mechanism dominates in all four cases under study. Our results are in perfect agreement with recent analytical studies of energy transfer processes in MHD turbulence by Verma et al. (2005), where velocity-to-velocity, magneticto-magnetic, velocity-to-magnetic, and magnetic-to-velocity energy transfers are found to be local in nonhelical MHD. These results are partially consistent with results of direct numerical simulations reported by Alexakis et al. (2005), where velocity-to-magnetic transfer has been found to be nonlocal, whereas the rest of the transfers have local character.

We find that the dominating transfer of fluctuations in turbulence in slow solar wind has similar character as in the case of Kolmogorov phenomenology describing turbulence in neutral fluids (Frisch, 1995), where according to the Richardson's cascade scenario, the energy transfer has local character in the wave vector space. This means that the energy at a given scale is transfered mainly to similar scales in solar wind turbulence.

Acknowledgements. We would like to thank the MAG and SWEPAM instruments teams (ACE spacecraft) for providing the magnetic field and bulk velocity data. This work has been supported by the Polish Ministry of Science and Higher Education through Grant No. N N202 412733.

Edited by: A. C. L. Chian

Reviewed by: two anonymous referees

\section{References}

Alexakis, A., Mininni, P. D., and Pouquet, A.: Shell-to-shell energy transfer in magnetohydrodynamics. I. Steady state turbulence, Phys. Rev. E, 72, 046301, 2005.

Biskamp, D.: Magnetohydrodynamic Turbulence, Cambridge: University Press, 2003

Bruno, R. and Carbone, V.: The Solar Wind as a Turbulence Laboratory, Living Reviews in Solar Physics, 2, 4, 2005.

Burlaga, L. F.: Multifractal structure of the interplanetary magnetic field - Voyager 2 observations near 25 AU, 1987-1988, Geophys. Res. Lett., 18, 69-72, 1991.

Burlaga, L. F.: Lognormal and multifractal distributions of the heliospheric magnetic field, J. Geophys. Res., 106, 15917-15928, 2001.

Davoudi, J. and Tabar, M. R.: Theoretical Model for the KramersMoyal Description of Turbulence Cascades, Phys. Rev. Lett., 82, 1680-1683, 1999.

Debliquy, O., Verma, M. K., and Carati, D.: Energy fluxes and shell-to-shell transfers in three-dimensional decaying magnetohydrodynamic turbulence, Phys. Plasmas, 12, 2309, 2005.

Friedrich, R. and Peinke, J.: Statistical properties of a turbulent cascade, Physica D, 102, 147-155, 1997a.

Friedrich, R. and Peinke, J.: Description of a Turbulent Cascade by a Fokker-Planck Equation, Phys. Rev. Lett., 78, 863-866, 1997b.

Frisch, U.: Turbulence. The legacy of A.N. Kolmogorov, Cambridge: University Press, 1995.

Goldstein, M. L. and Roberts, D. A.: Magnetohydrodynamic turbulence in the solar wind, Phys. Plasmas, 6, 4154-4160, 1999.

Goldstein, M. L., Roberts, D. A., and Matthaeus, W. H.: Magnetohydrodynamic Turbulence In The Solar Wind, Annu. Rev. Astron. Astrophys., 33, 283-326, 1995.

Hnat, B., Chapman, S. C., and Rowlands, G.: Intermittency, scaling, and the Fokker-Planck approach to fluctuations of the solar wind bulk plasma parameters as seen by the WIND spacecraft, Phys. Rev. E, 67, 056404, 2003.

Kishida, K., Araki, K., Kishiba, S., and Suzuki, K.: Local or Nonlocal? Orthonormal Divergence-Free Wavelet Analysis of Nonlinear Interactions in Turbulence, Phys. Rev. Lett., 83, 5487-5490, 1999.

Macek, W. M. and Szczepaniak, A.: Generalized two-scale weighted Cantor set model for solar wind turbulence, Geophys. Res. Lett., 35, L02108, doi:10.1029/2007GL32263, 2008.

Marsch, E. and Tu, C. Y.: Non-Gaussian probability distributions of solar wind fluctuations, Ann. Geophys., 12, 1127-1138, 1994, http://www.ann-geophys.net/12/1127/1994/.

Marsch, E., Tu, C.-Y., and Rosenbauer, H.: Multifractal scaling of the kinetic energy flux in solar wind turbulence, Ann. Geophys., 14, 259-269, 1996, http://www.ann-geophys.net/14/259/1996/.

Matthaeus, W. H. and Goldstein, M. L.: Measurement of the rugged invariants of magnetohydrodynamic turbulence in the solar wind, J. Geophys. Res., 87, 6011-6028, 1982.

McComas, D. J., Bame, S. J., Barker, P., Feldman, W. C., Phillips, J. L., Riley, P., and Griffee, J. W.: Solar Wind Electron Proton Alpha Monitor (SWEPAM) for the Advanced Composition Explorer, Space Sci. Rev., 86, 563-612, 1998.

Mininni, P., Alexakis, A., and Pouquet, A.: Shell-to-shell energy transfer in magnetohydrodynamics. II. Kinematic dynamo, Phys. Rev. E, 72, 046302, 2005. 
Pedrizzetti, G. and Novikov, E. A.: On Markov modelling of turbulence, J. Fluid Mech., 280, 69-93, 1994.

Pouquet, A., Frisch, U., and Leorat, J.: Strong MHD helical turbulence and the nonlinear dynamo effect, J. Fluid Mech., 77, 321354, 1976.

Renner, C., Peinke, J., and Friedrich, R.: Experimental indications for Markov properties of small-scale turbulence, J. Fluid Mech., 433, 383-409, 2001.

Risken, H.: The Fokker-Planck equation. Methods of solution and applications, Springer Series in Synergetics, Berlin, New York: Springer, 2nd ed., 1989.

Schwenn, R.: Large-Scale Structure of the Interplanetary Medium, in: Physics of the Inner Heliosphere I. Large-Scale Phenomena, edited by Schwenn, R. and Marsch, E., pp. 99-182, Berlin: Springer-Verlag, 1990.

Schwenn, R.: Solar Wind Sources and Their Variations Over the Solar Cycle, Space Sci. Rev., 124, 51-76, 2006.
Smith, C. W., L'Heureux, J., Ness, N. F., Acuña, M. H., Burlaga, L. F., and Scheifele, J.: The ACE Magnetic Fields Experiment, Space Sci. Rev., 86, 613-632, 1998.

Sorriso-Valvo, L., Carbone, V., Veltri, P., Consolini, G., and Bruno, R.: Intermittency in the solar wind turbulence through probability distribution functions of fluctuations, Geophys. Res. Lett., 26, 1801-1804, 1999.

Sorriso-Valvo, L., Carbone, V., Giuliani, P., Veltri, P., Bruno, R., Antoni, V., and Martines, E.: Intermittency in plasma turbulence, Planet. Space Sci., 49, 1193-1200, 2001.

Tu, C.-Y. and Marsch, E.: MHD structures, waves and turbulence in the solar wind: Observations and theories, Space Sci. Rev., 73, 1-210, 1995.

Verma, M. K., Ayyer, A., and Chandra, A. V.: Energy transfers and locality in magnetohydrodynamic turbulence, Phys. Plasmas, 12, 2307, 2005. 